\title{
Atrial pseudothrombus, a new radiological sign of right atrial roof bulging, caused by pericardial effusion, observed on chest CT
}

\begin{abstract}
Cardiac masses are rare findings, but they may have several etiologies, and classically can be divided as neoplastic or not-neoplastic. ${ }^{1}$ Due to the diversity of causes, the patient's clinical data such as age, symptoms, and previous pathological history are essential factors in guiding the diagnosis. The gold standard exam in the investigation of cardiac masses is currently the magnetic resonance exam, which presents high accuracy to differentiate thrombus tumors. ${ }^{2}$ Usually the echocardiography plays an important role being the first imaging study in evaluating the heart. ${ }^{2}$ However, with the increased use of imaging as a diagnostic method, the cardiac mass may be initially visualized on the chest CT scan. A satisfactory heart evaluation by chest CT is limited if it is non-triggered, mainly because of movement artifacts. However, if there any abnormalities are seen, hypotheses may be made based on patient's clinical story. In the case of atrial cardiac thrombi, multidetector computed tomography showed high sensitivity and specificity for diagnosis. ${ }^{3,4}$
\end{abstract}

Volume 6 Issue 6 - 2019

\author{
José Vitor Rassi Garcia,' Daphine Centola \\ Grassi,' Iona Grossman Pozzi, ${ }^{2}$ Claudia \\ Cosentino Gallafrio,' Henrique Manoel \\ Lederman' \\ 'Department of Radiology, GRAACC, Brazil \\ ${ }^{2}$ Department of Cardiology, GRAACC, Brazil
}

\begin{abstract}
Correspondence: José Vitor Rassi Garcia, Department of Radiology, GRAACC, Brazil, Tel +55 6I 98I30-8487,
\end{abstract}

Email josevitorrg@gmail.com

Received: October 18,2019 | Published: November 08, 2019

Keywords: cardiac masses, tumors, pseudotumor, echocardiography, thromboembolism

\section{Case report}

A 22-year-old female patient was admitted to the emergency room of an Oncologic center, with an acute onset of dry cough, hyaline runny nose, and sudden, severe, ventilatory-dependent chest pain, located in the parasternal region with shoulder irradiation, followed by dyspnea. On physical examination, associated findings are perioral and on the distal fingers' phalanges cyanosis. There was no history of fever, paresthesias, syncope, or other symptoms. Six years ago, in 2013, she had been diagnosed with 10th right costal arch Askin's tumor with pulmonary metastasis. Initially, she started receiving 1st line chemotherapy according to the Metastatic Ewing Sarcoma Protocol 2010, and then surgical approach for metastatic resection four right costal arches. So far, she has had three relapses, the first in 2016 for the left femur and lungs, in 2017 for the skullcap and 2018 for the right femur and right tibia. Since then, she has been receiving treatment according to an alternative chemotherapy protocol consisting by Topotecan $0.75 \mathrm{mg} / \mathrm{m}^{2}$ D1 to D5/CTX $250 \mathrm{mg} / \mathrm{m}^{2} \mathrm{D} 1$ to D5 every 21 days and radiotherapy. A year ago, she developed a chronic thrombosis of the left brachiocephalic vein.

Because of the acute onset of respiratory symptoms, considering the possible diagnosis of pulmonary thromboembolism, a chest CT angiography was performed. The CT findings demonstrated signs of previous surgical manipulation of the chest, the presence of left brachiocephalic vein thrombosis, associated with intense collateral circulation, more evident in the left side. There were also sparse consolidations of the pulmonary parenchyma, azygos vein ectasia and its arch and large pericardial effusion. In the right atrium, there was a hypoattenuating rounded formation with well-defined limits, adjacent to the lateral wall (Figure 1). Considering this set of imaging findings, the patient's clinical presentation and historical status, the hypothesis was that the image seen in the right atrial chamber could represent a large thrombus, with no signs of pulmonary thromboembolism. In conjunction with the medical team, it was decided to pursue further investigation and evaluation of the repercussions of the findings on cardiac function. The echocardiogram performed the day after the $\mathrm{CT}$, confirmed the extensive circumferential pericardial effusion, with signs of restriction of the ventricular filling, determining bulging of the right atrial roof and the right ventricular free wall, associated with tachycardia (108 bpm). There were no signs of thrombi or vegetations inside the cardiac chambers (Figure 2). As a consequence of that, a relief pericardiocentesis was done, draining $400 \mathrm{~mL}$, with significant improvement of the clinical symptoms. After that, whole-body magnetic resonance imaging was performed during hospitalization for oncological follow-up (Figure 3), confirming the resolution of the pericardial effusion and no evidence of endocardial tumors.

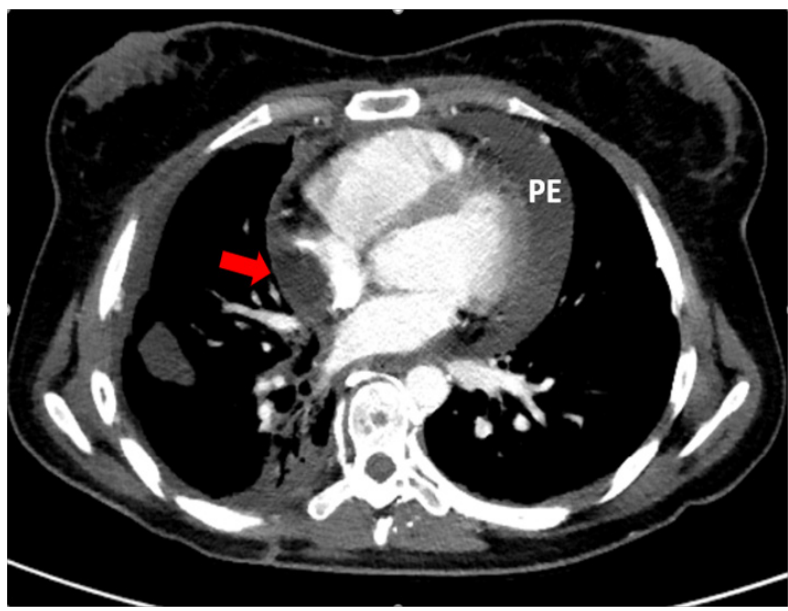

Figure I Chest CT scan demonstrating a large pericardial effusion (PE) and a hypoattenuating rounded formation with well-defined limits, adjacent to the lateral wall, in the right atrium (red arrow).

\section{Discussion}

Cardiac tumors, as already briefly mentioned, have several differential diagnoses, often presenting different imaging characteristics on echocardiographic examination, and especially on magnetic resonance imaging (Table 1). However, on nontriggered chest computed tomography examination, the diagnosis of cardiac masses is often incidental, and is less accurate when compared to the previously mentioned methods, ${ }^{1,5}$ mainly because of 
movement artifacts, which may even create false images and induce misinterpretation. ${ }^{6}$ For example, the most common type of "heart tumor" is pseudotumor, such as heart thrombi and normal anatomic variants. ${ }^{6}$

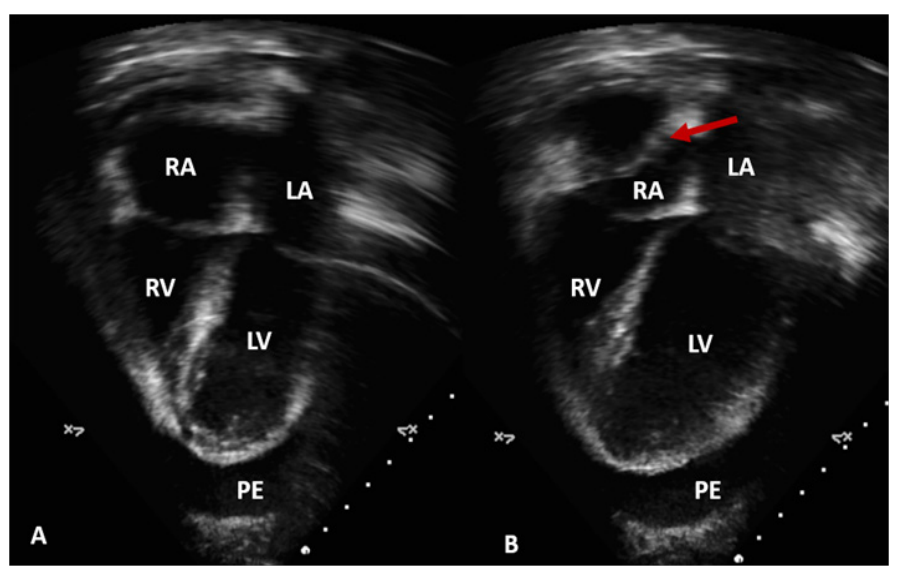

Figure 2 Echocardiogram, Apical 4 chamber view. (A) Images of the four cardiac chambers during ventricular systole, with right atrial cavity within normal range. (B) Bulging of the right atrial wall ceiling (red arrow) during final ventricular diastole. RA, right atrium; LA, left atrium; RV, right ventricle; LV, left ventricle; $\mathrm{PE}$, pericardial effusion

In the case presented, we observed a large pericardial effusion and a well-defined hypoattenuated image inside the right atrium that mimicked a thrombus, which was corroborated by the patient's clinical presentation and previous diagnostic history. The echocardiogram, performed in sequence, showed a free right atrial chamber, without

Table I The table summarizes normal structures in the atria that can simulate pathology and how to avid misinterpretations

\begin{tabular}{lll}
\hline Cardiac mass & & \\
\hline Pseudo Tumor & Benign & Malignant \\
Thrombus & Myxoma & Angiosarcoma \\
Pericardial cyst & Lipoma & Rhabdomyosarcoma \\
& Fibroma & Lymphoma \\
& Rhabdomyoma & Metastasis \\
\hline
\end{tabular}

Table 2 Normal structures in the ventricles that can mimic diseases and appropriate investigations to prevent mismterpretations

\begin{tabular}{lll}
\hline $\begin{array}{l}\text { Cardiac } \\
\text { chambers }\end{array}$ & Normal structures & Mimickers \\
\hline Right Atrium & Proeminet crista terminalis & Thrombus or tumor \\
& Proeminet pectinatemuscle & Thrombus or tumor \\
& $\begin{array}{l}\text { Proeminet Eustachian and } \\
\text { thebasian valves }\end{array}$ & Thrombus or tumor \\
\hline
\end{tabular}

On the retrospective evaluation of the tomographic exam, some signs could be used to indicate that the image of the "pseudotumor" was not a true thrombus; such the hypoattenuated image had fat density, ranging from -10 to $-20 \mathrm{UH}$. The density assessment is an essential indicator for differentiating a true thrombus, which usually tumors or pseudotumors inside in association with a large pericardial effusion described on the tomography. However, the echocardiogram showed signs of tamponade, ${ }^{7}$ filling restriction of the ventricular artery and bulging of the right atrial roof during diastole. The first description of this atrial wall abnormality was in the echocardiographic study in $1983,{ }^{8}$ which could be observed on the non-triggered chest CT scan of the patient in this report, mimicking an atrial pseudotumor, an entity hitherto not described in the literature for chest computed tomography studies. The rarity of this finding on tomography studies is because this is an image secondary to the atrial movement. There must be a right timing to the image acquisition during atrial diastole. That is why this tomographic sign was affectionately referred to as the "Damasceno pseudothrombus" in honor of the technician who performed the examination, at which time the external pressure of the pericardial effusion becomes more significant than the right atrial internal pressure, causing the bulging from your ceiling. When this bulging occurs for more than $1 / 3$ of the ventricular systole time, the sensitivity and specificity for cardiac tamponade are close to $100 \%{ }^{7}$

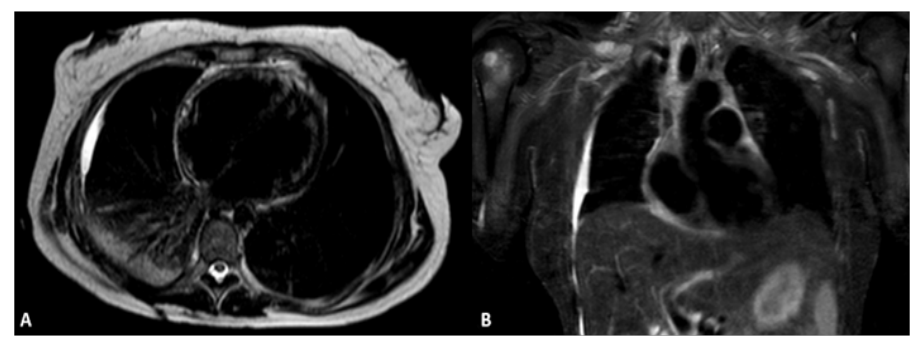

Figure 3 Total-body MRI confirming the resolution of the pericardial effusion and no evidence of endocardial tumors.

has higher density, between 20 and $50 \mathrm{UH} .{ }^{9}$ However, it is crucial to emphasize that the most common etiologies of intracardiac lesions may reveal, on chest $\mathrm{CT}$, as fat-density, ${ }^{10,11}$ namely: lipomas (benign mass), liposarcomas (malignant mass), hypertrophy, lipomatosis of the interatrial septum (non-neoplastic). Other differential diagnoses of imaging pitfalls such as anatomical variants and other cardiac motion artifacts that mimic disease are still to be considered, ${ }^{5}$ in especial the right atrium because of the prominence of the pectineus muscle, the terminal crest and the Eustachio and Thebesian valves, which can mimic thrombi or tumors on $\mathrm{CT}$, and should proceed to diagnostic elucidation with magnetic resonance imaging or echocardiogram (Table 2). ${ }^{5}$ A recent study demonstrated that a two-phase cardiac CTA showed $100 \%$ of sensitivity and $98 \%$ of specificity compared to transesophageal echocardiography for the assessment of thrombi located in the left atrial appendage in patients who suffered ischemic stroke. ${ }^{11}$

Imaging acquisition technique on cardiac CTA is extremely specific, which allows a great evaluation of the heart chambers. Cardiac CTA's specifications include usually the patient is in a bradycardic state $(<60 \mathrm{bpm})$, imaging acquisition method is coupled to the electrocardiogram and all images are reconstructed on ventricular diastole. Because of that, if the case presented was scanned according to the cardiac CTA protocol, probably, the pseudo lesion wouldn't have appear. ${ }^{12}$

\section{Conclusion}

The case presented shows an oncologic patient with multiple comorbidities and previous treatments, including previous thrombosis 
events. All of them counts for cardiotoxic and therefore triggers cardiac alterations. ${ }^{13}$ In this scenario, with acute onset of respiratory distress associated with the imaging findings in the CTA, the hypothesis of atrial thrombus (hypoattenuation within the right atrium, abundant thoracic collateral circulation, and azygos vein ectasia) was strongly considered. The echocardiography examination was an important tool for the diagnosis. However, we demonstrate the importance of a specific oriented CTA in order to make any differential diagnoses for cardiac entities.

\section{Acknowledgments}

None.

\section{Ethical consent}

None.

\section{Funding}

None.

\section{Conflicts of interest}

Author declares that there is no conflict of interest.

\section{References}

1. Kassop D, Donovan M, Cheezum M, et al. Cardiac masses on cardiac CT: a review. Curr Cardiovasc Imaging Rep. 2014;7(8):9281.

2. Buckley O, Madan R, Kwong R, et al. Cardiac masses, part 1: imaging strategies and technical considerations. AJR Am J Roentgenol. 2011;197:W837-W841.

3. Wu X, Wang C, Zhang C, et al. Computed tomography for detecting left atrial thrombus: a meta-analysis. Arch Med Sci. 2012;8(6):943-951.
4. Tan CH, Pua U, Chia PL. Multidetector row CT diagnosis of an infected right atrial thrombus following repeated dialysis catheter placement. $\mathrm{Br}$ J Radiol. 2009;82(984):e240-e242.

5. Terpenning S, White CS. Imaging pitfalls, normal anatomy, and anatomical variants that can simulate disease on cardiac imaging as demonstrated on multidetector computed tomography. Acta Radiol Short Rep. 2015;4(1):2047981614562443.

6. Motwani M, Kidambi A, Herzog BA, et al. MR imaging of cardiac tumors and masses: a review of methods and clinical applications. Radiology. 2013;268(1):26-43.

7. Pérez-Casares A, Cesar S, Brunet-Garcia L, et al. Echocardiographic Evaluation of Pericardial Effusion and Cardiac Tamponade. Front Pediatr. 2017;5:79.

8. Gillam LD, Guyer DE, Gibson TC, et al. Hydrodynamic compression of the right atrium: a new echocardiographic sign of cardiac tamponade. Circulation. 1983;68(2):294-301.

9. Scheffel H, Baumueller S, Stolzmann P, et al. Atrial myxomas and thrombi: comparison of imaging features on CT. AJR Am J Roentgenol. 2009;192(3):639-645.

10. Hur J, Kim YJ, Lee HJ, et al. Left atrial appendage thrombi in stroke patients: detection with two-phase cardiac CT angiography versus transesophageal echocardiography. Radiology. 2009;251(3):683-690.

11. Kim EY, Choe YH, Sung K, et al. Multidetector CT and MR imaging of cardiac tumors. Korean J Radiol. 2009;10(2):164-175.

12. Kalisz K, Buethe J, Saboo SS, et al. Artifacts at cardiac CT: physics and solutions. Radiographics. 2016;36(7):2064-2083.

13. Zhu Q, Kirova YM, Cao L, et al, Cardiotoxicity associated with radiotherapy in breast cancer: A question-based review with current literatures. Can Treatment Rev. 2018;68:9-15. 